\title{
PROJETO PRELIMINAR DA GEOPOLITICA DOS MUSEUS DA DIASPORA AFRICANA NO BRASIL
}

\author{
Rafael Sanzio Araújo dos Anjos \\ Izabella C. de A. dos Anjos \\ Richard Denis
}

\section{Revista}

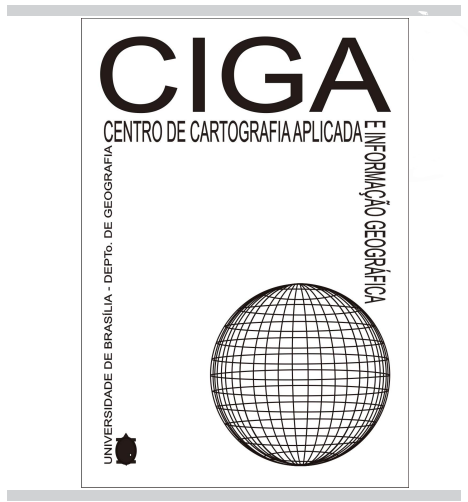

Revista Eletrônica:

Tempo - Técnica - Território, V.9, N.3 (2018), 21:30

ISSN : $2177-4366$
Como citar este ar tigo:

Sanzio, R. Richard Denis, Izabella C. de A. dos Anjos. PROJETO

PRELIMINAR DA GEOPOLITICA DOS MUSEUS DA DIASPORA AFRICANA

NO BRASIL. Revista Eletrônica: Tempo - Técnica - Território, v.9, n.3 (2018), p. 21:30 ISSN: 2177-4366.

Disponível em:

http://periodicos.unb.br/index.php/ciga/issue/view/1916

Este obra está licenciado com uma Licença Creative Commons Atribuição-NãoComercial 4.0 Internacional. 


\section{PROJETO PRELIMINAR DA GEOPOLITICA DOS MUSEUS DA DIASPORA AFRICANA NO BRASIL}

\section{8}

\section{NOTA TÉCNICA}

Rafael Sanzio Araújo dos Anjos (*), Richard Denis, Izabella C. de A. dos Anjos (**) Email:cartografia@unb.br Site:www.unb.br

(*) Geógrafo, Doutor em Informações Espaciais Pós-Doutorado Cartografia Étnica. Prof. Titular da Universidade de Brasília / Diretor do Centro de Cartografia Aplicada e Informação Geográfica do Depto. de Geografia - UnB

(**) Pesquisadora Colaboradora do CIGA e Estagiários membros do Centro de pesquisa na UnB

RESUMO : A Cartografia da Geopolítica dos Museus da Diáspora Africana no Brasil é um projeto desenvolvido pelo CIGA em parceria com a professora de museologia Deborah Santos e dedica-se a representar cartograficamente a distribuição espacial dos museus com acervos da disporá africana no território Brasileiro. Além de expor de forma cartográfica a localização de tais acervos o projeto também compila informações relevantes sobre os itens dos acervos, as atividades desenvolvidas nos museus e um pouco de sua historia e relevância para a comunidade local.

ABSTRACT: The Cartography of Geopolitics of Museums of the African Diaspora in Brazil is a project developed by CIGA in partnership with a professor of museology called Deborah Santos. The project dedicate itself to represent cartographically the spatial distribution of museums with goods of interest of African-Brazilian nature. In addition to mapping the location of such collections, the project also compiles relevant information about the items in the collections, the activities developed in the museums, and a little of its history and relevance to the local community.

\section{1 - A CARTOGRAFIA, O ORDENAMENTO DO TERRITÓRIO.}

A informação espacial referente à forma de ocupação que se processa no território constituiu uma das mais estratégicas para responder e informar sobre as inúmeras indagações do que está acontecendo, do que pode acontecer e do que aconteceu no espaço geográfico. Nesse sentido, o mapeamento das ocupações e dos espaços como 
ferramenta de armazenamento e comunicação de dados geográficos têm experimentado significativos avanços nas fontes geradoras das informações, principalmente nas últimas décadas, com os produtos de sensoriamento remoto de última geração e as tecnologias computacionais para manipulação e referencia mento da informação territorial. Como ponto inicial do desenvolvimento da pesquisa, abordamos brevemente os pressupostos instrumentais e metodológicos utilizados na confecção da cartografia temática da Geopolítica da Diáspora Africana no Brasil. Com este trabalho buscamos contribuir efetivamente para a ampliação do conhecimento sobre os acervos da diáspora africana espalhados no território nacional brasileiro, evidenciando a geopolítica dos museus africanos e sua distribuição espacial no âmbito nacional.

\section{2 - O CIGA.}

Não podemos perder de vista que a Universidade de Brasília (UnB) consolidou-se como uma das melhores instituições de ensino, pesquisa e extensão do Brasil, reconhecida pela qualidade na sua produção acadêmica e pela elevada qualificação de seu corpo docente. Seguindo as suas referências básicas, o Departamento de Geografia e o Programa de Pós Graduação em Geografia da UnB constituíram, em 1999, o Centro de Cartografia Aplicada e Informação Geográfica (CIGA), com o propósito de dar maior amplitude nos projetos e parcerias em andamento, a partir da integração da educação, pesquisa e atendimento às demandas da sociedade civil. O CIGA tem uma estrutura física organizada com equipamentos básicos para o desenvolvimento de atividades direcionadas para o manuseio de ferramentas geográficas voltadas para a educação geográfica e o planejamento-gestão do território. É importante destacar que os sistemas de informação territorial tem presenciado uma forte difusão de dados e tornado acessíveis outras possibilidades de representação do espaço geográfico. Por outro lado, as demandas para compreensão e resolução das complexas questões da dinâmica da sociedade são crescentes e a cartografia se confirma como uma das disciplinas melhor colocadas para responder e informar sobre o que aconteceu, o que está acontecendo de fato e o que pode acontecer no território, ou seja, as representações cartográficas possibilitam trabalhar com o passado, o presente e o futuro próximo do território. Após estes ano de atividades, o CIGA consolida a sua vocação de referência local, regional e nacional na produção técnica de aplicabilidades e alcance social das ferramentas geográficas ligadas à cartografia, sensoriamento remoto e sistemas de informação geográfica.

\section{3 - A REPRESENTAÇÃO CARTOGRÁFICA DA GEOPOLÍTICA DOS ACERVOS DA DIÁSPORA.}

Neste documento esta contido o resumo das operações técnicas deflagradas para a elaboração do projeto da Geopolítica dos museus da diáspora africana no Brasil, desenvolvido em colaboração com a professora Deborah Santos, do curso de Museologia da Universidade de Brasília, referente a museus e acervos que concentram obras da memoria cultural da disporá Africana. Em um primeiro momento será explicado o processo de atualização pelo qual as fichas passaram entre outubro e 
dezembro de 2017, em seguida apresentamos as mudanças metodológicas que tivemos que adotar para sanar alguns problemas técnicos referentes à numeração dos museus e a mescla dos dados que possuíamos com os dados coletados por Deborah Santos. Mais a frente esta um modelo de estrutura padrão das fichas, que serve para exemplificar como as informações estarão distribuídas em todas as fichas. Ademais, expomos o procedimento empregado para referenciar espacialmente os museus nas fichas, com suas respectivas coordenadas e imagens aéreas. Por fim, trazemos o mapa final elaborado e seu respectivo metadado.

\section{4 - ATUALIZAÇÃO DAS FICHAS}

As fichas utilizados no projeto Geopolítica dos Museus com Acervo Africano no Mundo foram utilizada como base fundamental para a pesquisa em conjunto com a base de dados fornecidas pela professora Deborah Santos. Os museus catalogados foram pesquisados e georeferenciados, de forma que obtivemos e fichamos dados acerca da historia e atividades desenvolvidas nos museus, além de sua localização espacial. Devido à fusão de dados e de dois projetos de pesquisa iniciados de forma independente foi necessário renumerar todas as fichas segundo o novo padrão de numeração. 


\section{5 - ESTRUTURAÇÃO DAS FICHAS}

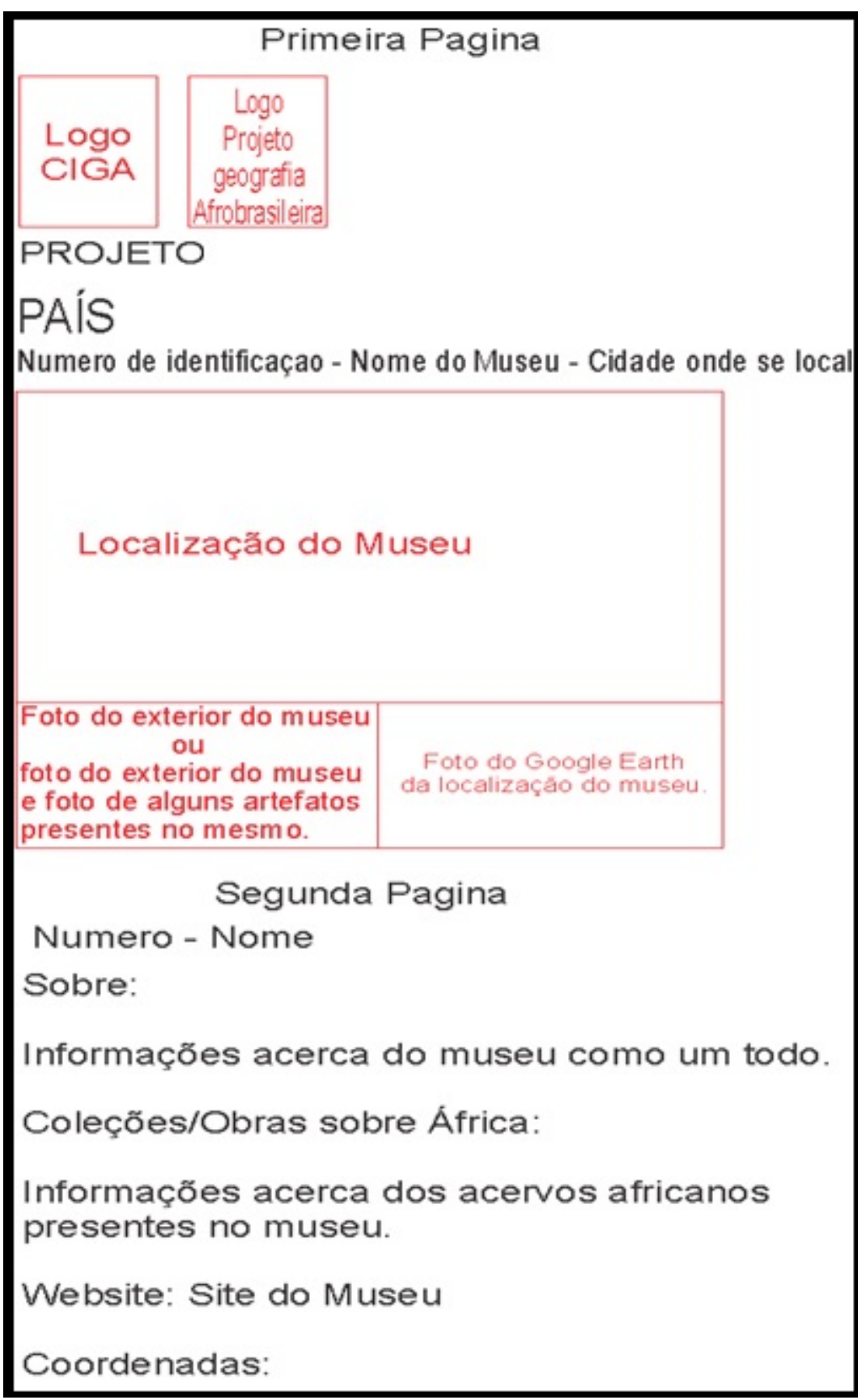

(Modelo de ficha esquematizada)

\section{6 - REFERENCIAMENTO ESPACIAL DOS MUSEUS}

As fichas dos museus passaram por um processo de atualização de informações e georreferenciamento espacial. Os museus foram localizados através do Google Maps e do Google Earth e os dados de suas posições geográficas foram adicionadas as fichas. Uma lista base de museus e seus dados foi fornecida pela professora Deborah. Em um primeiro momento os museus foram localizados no Google Maps, uma foto aérea ou 
mapa virtual do museu e de suas proximidades foi adicionado às fichas que não a possuem. Nas fotos aéreas foram “hiperlinkados”' os endereções eletrônicos referentes a localização do museu no Google Maps. Além disso, ao final das fichas estão sendo adicionadas as Coordenadas dos museus.

Esse procedimento é a primeira fase da obtenção de dados geográficos dos museus e integração dessas informações online com as fichas. Segue abaixo ilustração de todas as etapas.

6.1 - A obtenção da localidade no Google Maps pode ser vista na imagem 1.

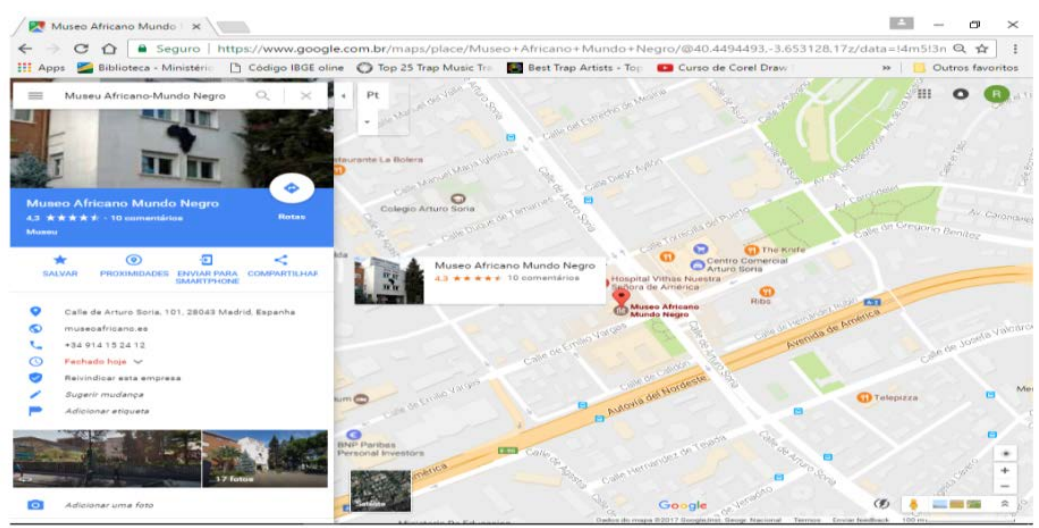

\section{(Imagem 1)}

6.2 - Adição de hiperlink de comunicação entre a ficha e a localização do museu, pode ser vista nas imagens 2 e 3 .

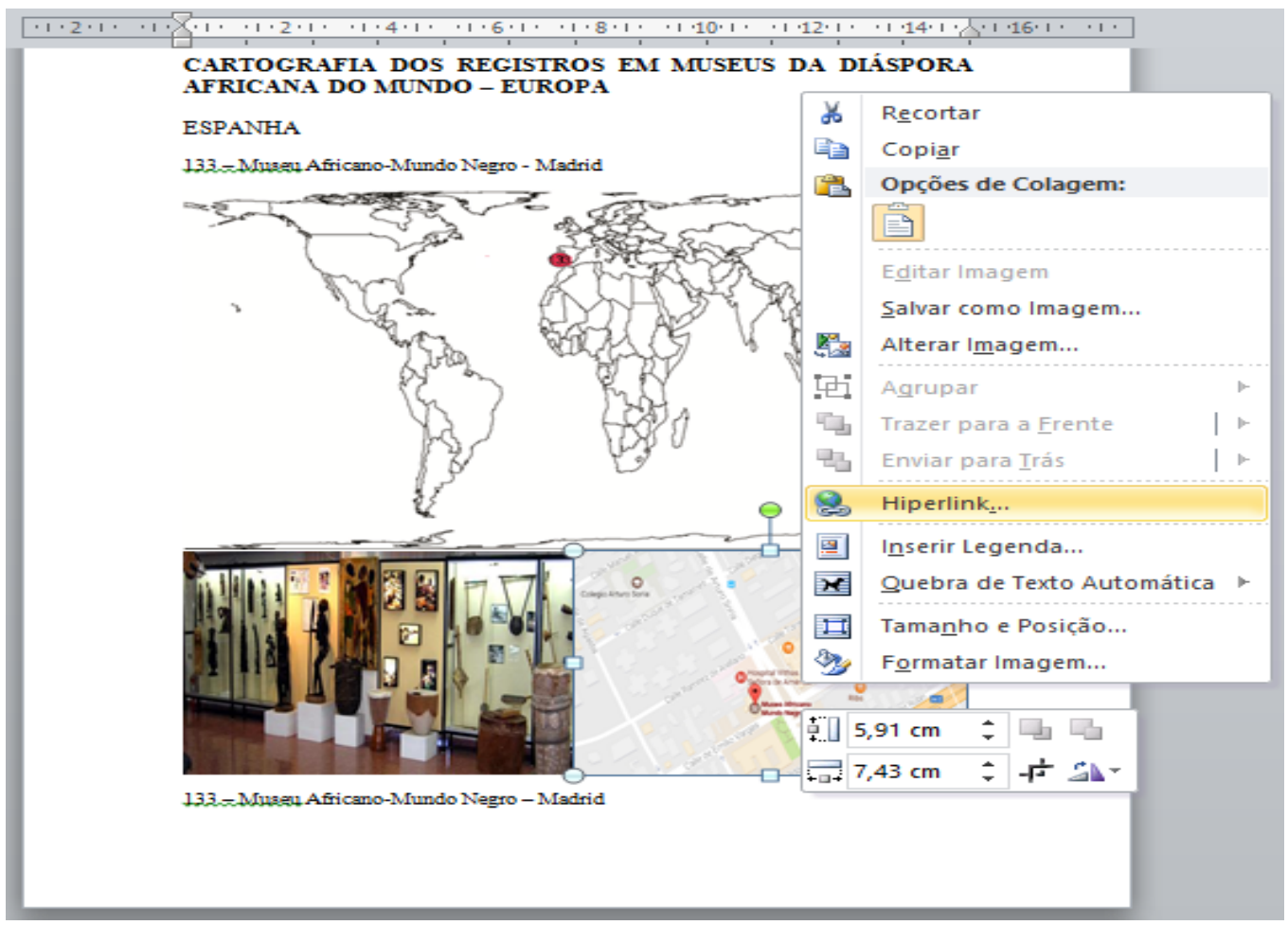

(Imagem 2) 


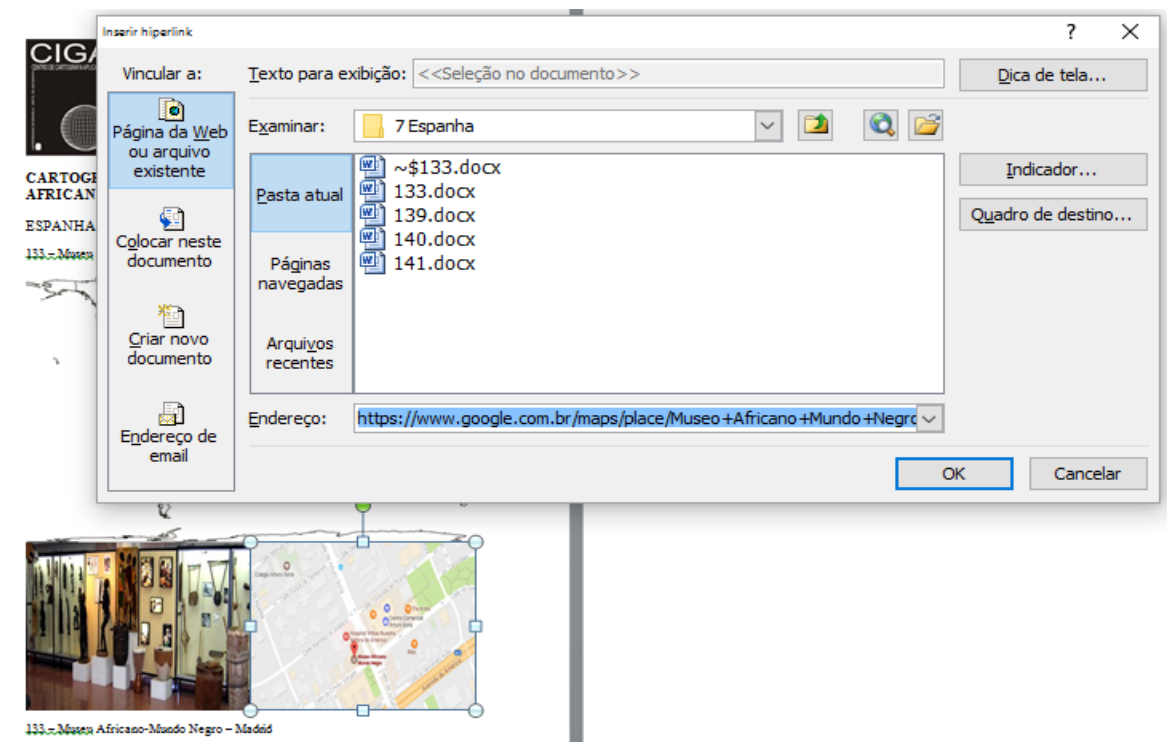

(Imagem 3)

6.3 - Adição das coordenada obtidas por meio do Google Earth, o processo pode ser visto na imagem 4.

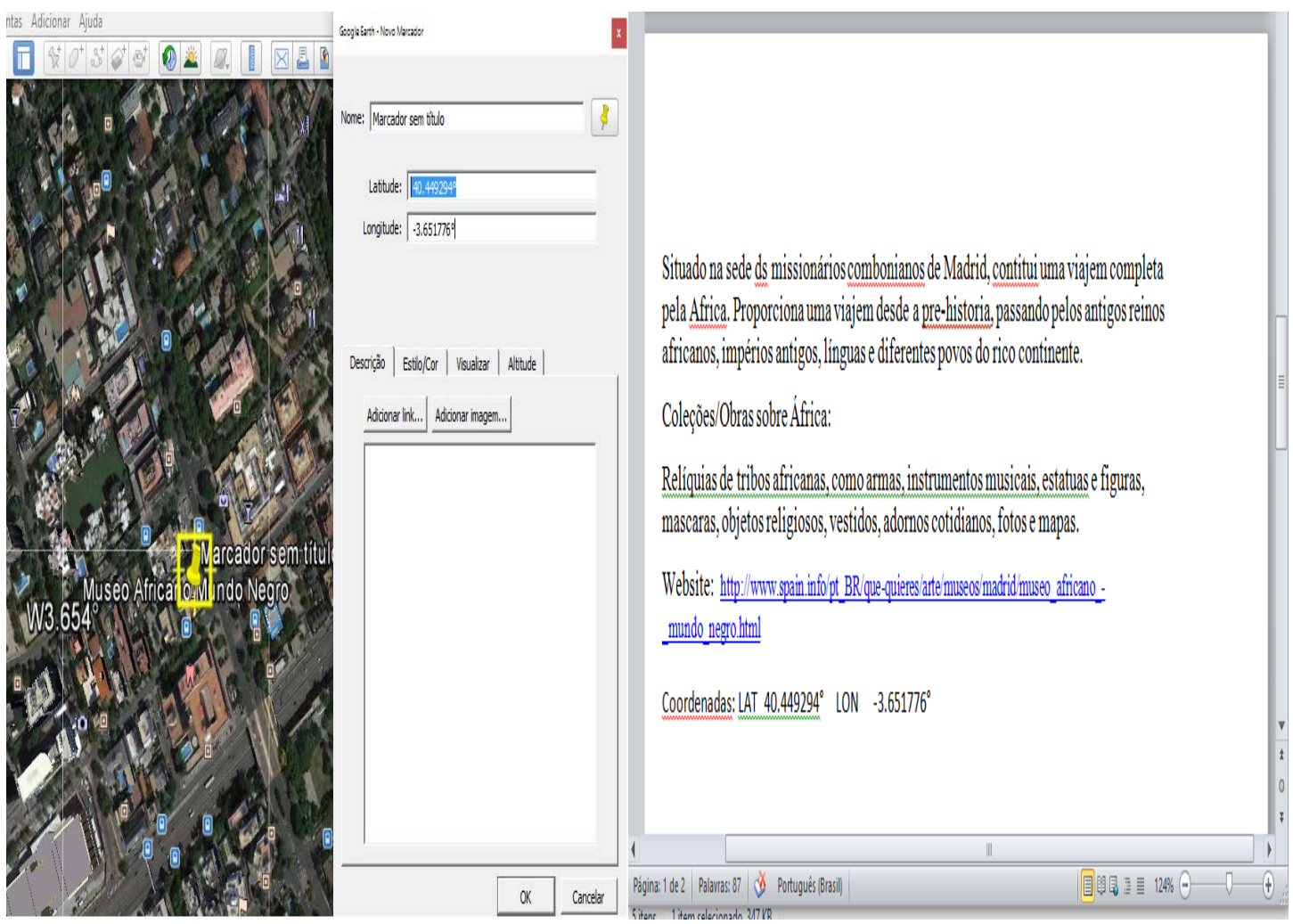

(Imagem 4) 


\section{7 - LISTA DOS MUSEUS CONTEMPLADOS PELA PESQUISA}

BR_AL_01 - Parque Memorial Quilombo dos Palmares

BR_BA_02 - Museu Afro Cultural Oyá Ní

BR_BA_03 - Museu Último Quilombo

BR_BA_04 - Museu Afro Omon Ajagunan

BR_BA_05 - Museu Comunitário Mãe Mirinha de Portão

BR_BA_06 - Museu Ilê Ohun Lailai

BR_BA_07 - Memorial Lajoumim - Terreiro Pilão de Prata

BR_BA_08 - Memorial Mãe Menininha do Gantois

BR_BA_09 - Museu Nacional da Cultura Afro-brasileira

BR_BA_10 - Casa de Cultura do Benin

BR_BA_11 - Museu Afro-Brasileiro

BR_BA_12 - Casa da Angola

BR_BA_13 - Memorial Kisimbiê

BR_BA_14 - Memorial das Baianas do Acarajé

BR_BA_15 - Casa do Samba - Centro de Referencia do Samba de Roda do Recôncavo da Bahia

BR_BA_16 - Memorial da Irmandade de N. Senhora da Boa Morte

BR_BA_17 - Memorial Unzo Tombenci Neto

BR_CE_18 - Museu Senzala Negro Liberto

BR_CE_19 - Museu Memorial da Liberdade

BR_SE_20 - Museu Afro-Brasileiro de Sergipe

BR_PB_21 - Memorial Jackson do Pandeiro

BR_PE_22 - Museu da Abolição

BR_PE_23 - Museu do Gonzagão

BR_PE_24 - Memorial Luiz Gonzaga

BR_PE_25 - Museu de Artes Afro-Brasil Rolando Toro

BR_RN_26 - Museu Escravo Jacó

BR_MA_27 - Cafua das Mercês (Museu do Negro) 
BR_MA_28 - Memorial da Balaiada

BR_MG_29 - Centro de Referência da Cultura Negra

BR_MG_30 - Museu do Escravo

BR_MG_31 - Museus dos Quilombos e Favelas Urbanos

BR_ES_32 - Museu Capixaba do Negro

BR_RJ_33 - Memorial Afro-Valenciano Padre João José da Rocha

BR_RJ_34 - Museu do Negro

BR_RJ_35 - Museu Ode Gbomi

BR_RJ_36 - Museu de Favela

BR_RJ_37 - Museu da Maré

BR_RJ_38 - Museu do Escravo

BR_RJ_39 - Centro Cultural Cartola

BR_SP_40 - Òsun Ìya Oke Ile Afro-Brasileiro Ode Lorecy

BR_SP_41 - Museu Afro Brasil

BR_SP_42 - Museu dos Escravos

BR_SP_43 - Museu da Cultura Africana e Negritude Brasileira

BR_SP_44 - Museu do Negro de Campinas

BR_SP_45 - Instituto Cultural Babá Toloji

BR_RS_46 - Museu Treze de Maio

BR_PE_47 - Cybermuseu Pernambuco

BR_BA_48 - Cybermuseu Bahia

BR_MA_49 - Cybermuseu Maranhão

BR_RJ_50 - Cybermuseu Rio de Janeiro

BR_MT_51 - Cybermuseu Mato Grosso

BR_BA_52 - Ilê Axé Opó Afonjá

BR_SE_53 - Museu afro-brasileiro de Laranjeiras

BR_GO_54 - Espaço de Memória do Quilombo do Mesquita

BR_PA_55 - Museu Paraense Emilio Goeldi

BR_MA_56 - Colações Afro Maranhenses - Patrimonios dos Terreiros Mina-Jeje e Mina-Nagô e das festas

Revista Eletrônica: Tempo - Técnica - Território, V.9, N.3 (2018), 21:30 ISSN: 2177-4366 
BR_CE_57 - Museu Arthur Ramos

BR_RN_58 - Museu Câmara Cascudo

BR_PE_59 - Fundação Gilberto Freyre

BR_PE_60 - Museu do Estado

BR_PE_61 - Museu da Abolição

BR_PE_62 - Museu do Homem do Nordeste

BR_AL_63 - Museu Théo Brandão de Antropologia e Folclore

BR_AL_64 - Coleção Perseverança de Alagoas - Instituto Histórico e Geográfico de Alagoas

BR_SE_65 - Museu Afro-Brasileiro de Sergipe

BR_BA_66 - Museu Afro-Brasileiro

BR_BA_67 - Candomblé de Salvador - Coleção do Instituto Geográfico e Histórico da Bahia

BR_BA_68 - Instituto Feminino da Bahia

BR_BA_69 - Museu Carlos Costa Pinto - Pencas de Balangandâs

BR_RJ_70 - Museu Nacional

BR_RJ_71 - Museu do Folclore Edison Carneiro

BR_RJ_72 - Museu Nacional de Belas Artes

BR_SP_73 - Museu de Arqueologia e Etnologia

BR_SP_74 - Pinacoteca do Estado

BR_SP_75 - MASP

BR_SP_76 - Museu AfroBrasil

\section{8 - Mapa geral do Brasil com a localização dos diversos acervos da diáspora.}

O mapa que segue a abaixo foi elabora com a finalidade de condessar, em um único produto cartográfico, a localização de todos os diferentes acervos da diáspora dispersos no território brasileiro. Os museus estão identificados segundo a natureza de suas características, distribuídos entre museus públicos, privados, com vinculo com alguma instituição religiosa, cybermuseus e aqueles sediados em instituições de ensino superior. 

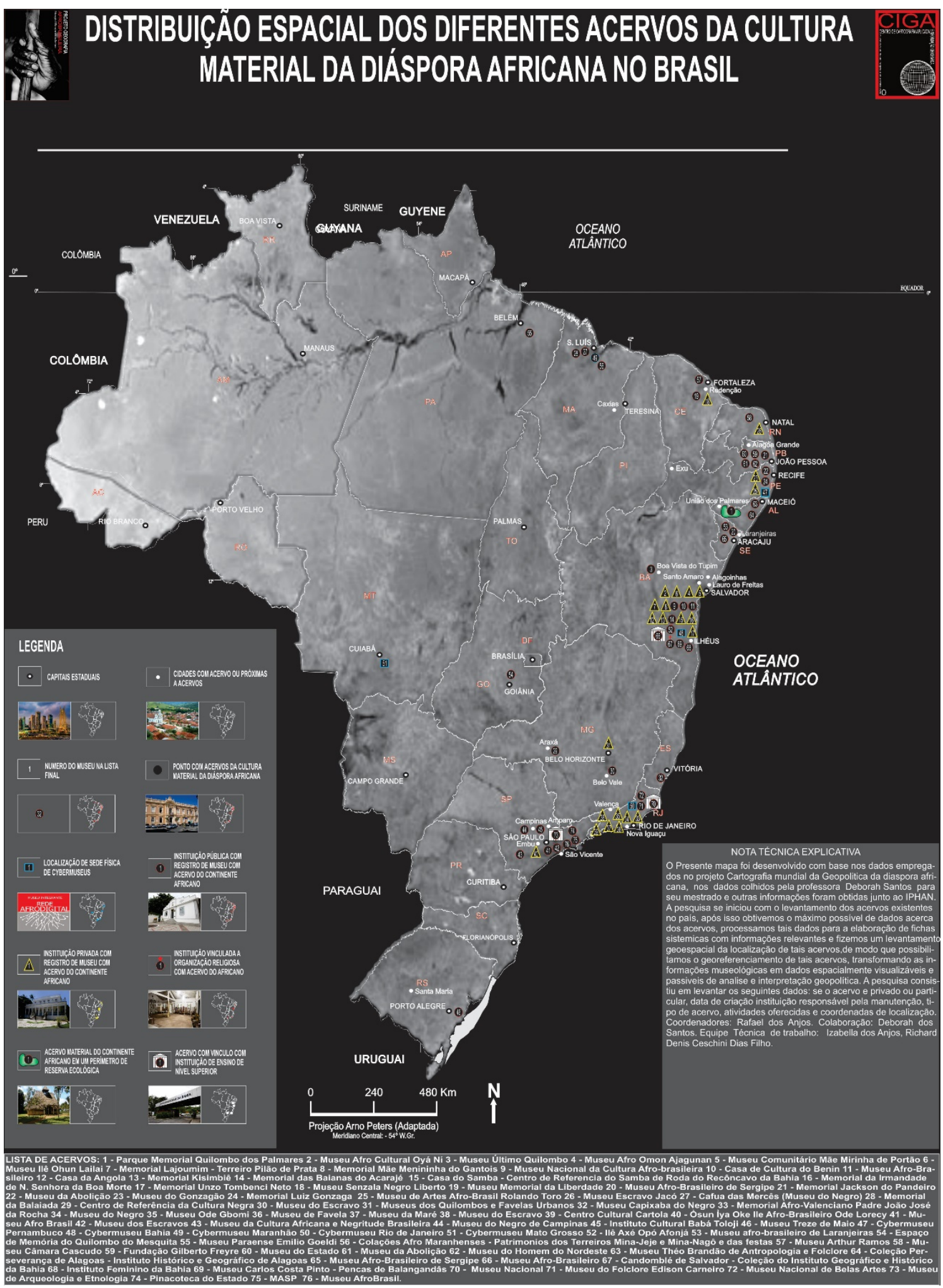

Metadados:

http://164.41.109.9:8080/geonetwork/srv/por/catalog.search\#/metadata/53497326-4c2c4460-9aea-15d13a7d2a6c 